\title{
REVISIÓN SISTEMÁTICA DEL GÉNERO BERBEROMELOE BOLOGNA, 1988 (COLEOPTERA, MELOIDAE) Y DIAGNOSIS DE UN ENDEMISMO IBÉRICO OLVIDADO
}

\author{
M. García-París $(*)$
}

\begin{abstract}
RESUMEN
El examen de 376 individuos de Berberomeloe, un género ibero-magrebí de Meloidae descrito por Bologna (1988), revela que el género está compuesto por dos especies: B. majalis (Linnaeus, 1758) y B. insignis (Charpentier, 1818). La distribución geográfica de $B$. majalis se extiende por la mayor parte de España y Portugal en la Península Ibérica, por una porción del sur de Francia, y a lo largo de las regiones septentrionales de Marruecos, Argelia y Túnez en el noroeste de Africa. B. insignis es una especie muy poco conocida, endémica de una estrecha franja costera del sureste español de las provincias de Granada, Almería y Murcia. Aunque B. insignis ha sido generalmente incluida en la sinonimía de B. majalis, sus rasgos morfológicos, claramente distintivos y entre los que se incluyen antenas con los segmentos IV al X alargados y particularmente dentados en el macho, ángulos anteriores del pronoto salientes y coloración cefálica con las sienes de color rojo-anaranjado, favorecen su reconocimiento a nivel específico.
\end{abstract}

Palabras clave: Coleoptera, Meloidae, Berberomeloe, B. insignis, B. majalis, Sistemática, Morfología.

\begin{abstract}
Systematics of the genus Berberomeloe Bologna, 1988 (Coleoptera, Meloidae) and diagnosis of an overlooked Iberian endemism
\end{abstract}

A review of 376 individuals of Berberomeloe, an African-Iberian genus of Meloidae described by Bologna (1988), reveals that the genus is composed of two species: B. majalis (Linnaeus, 1758) and B. insignis (Charpentier, 1818). The geographic range of $B$. majalis covers most of Spain and Portugal in the Iberian Peninsula, a portion of Southern France, and the northern regions of Morocco, Algeria and Tunisia in northwestern Africa. B. insignis is a little known taxon endemic of a narrow coastal zone in southeastern Spain, along the provinces of Granada, Almería and Murcia. Although B. insignis has been usually included as a synonym of B. majalis, its distinctive morphology, which includes among other traits, elongate IV to X antennae segments markedly dentate in males, prominent anterior angles of the pronotum, and a red-orange coloration on the posterior portions of the head, favour its recognition at the species level.

Key words: Coleoptera, Meloidae, Berberomeloe, B. insignis, B. majalis, Taxonomy, Morphology.

\footnotetext{
* Museo Nacional de Ciencias Naturales. CSIC. José Gutiérrez Abascal, 2. 28006 Madrid. España
} 


\section{Introducción}

El género Berberomeloe fue creado por Bologna (1988) para B. majalis (L.), una especie iberomagrebí tradicionalmente incluida en el género Meloe (tribu Meloini) por sus características morfológicas externas, pero cuya morfología larvaria no deja lugar a dudas sobre su inclusión entre los Lyttini (Górriz, 1882; MacSwain, 1956; Bologna, 1988). A pesar de que $B$. majalis es una especie llamativa y relativamente abundante en la mayor parte de la Península Ibérica su situación taxónomica ha sido objeto de numerosas controversias y ha sido incluida en tres géneros distintos pertenecientes a dos tribus bien diferenciadas. La especie fue descrita por Linneo (1758) en el género Meloe donde ha sido mantenida por diversos autores (Reitter, 1911; Fuente, 1933; Porta, 1934; Kaszab, 1969), a pesar de que los trabajos de Górriz $(1878 ; 1882)$ y Cros $(1912 ; 1940)$ claramente apuntaban sus afinidades con miembros de la tribu Lyttini. MacSwain (1956), seguido por Pinto y Selander (1970), transfirieron la especie a la tribu Lyttini, pero incluyendola en el género Trichomeloe Reitter, hasta que finalmente Bologna (1988) creó el género Berberomeloe para $B$. majalis manteniendolo dentro de los Lyttini. La morfología externa de Berberomeloe, con el abdomen enormemente desarrollado, áptera y de aspecto general muy similar a las especies del género Meloe han propiciado el que la especie haya sido incluida dentro de la tribu Meloini en muchos trabajos de ámbito regional.

Tanto en su antigua posición dentro de los Meloini, como actualmente entre los Lyttini, los meloideos de este género han recibido poca atención desde un punto de vista taxonómico y, exceptuando el trabajo monográfico de Bologna (1988), la mayor parte de los estudios anteriores han sido enfocados hacia su biología (Mulsant, 1857; Górriz, 1878, 1881, 1882; Cros, 1912, 1913, 1928, 1940; Ruiz et al., 1994). Aunque existen descripciones de variaciones individuales concernientes al desarrollo relativo de los élitros o a la presencia de manchas rojas tegumentarias (Olivier, 1792; Lucas, 1849; Reitter, 1872) la variabilidad geográfica de Berberomeloe no ha sido estudiada y, dentro del grupo, únicamente se reconocía la especie $B$. majalis (L.) (Bologna, 1991). Sin embargo, algunas poblaciones del sureste ibérico fueron consideradas anteriormente como una especie independiente bajo la denominación de Meloe insignis Charpentier, 1818, tal como lo atestiguan los trabajos de Górriz (1882), Rodríguez López-Neyra
(1914) y las etiquetas de identificación que L. Pérez Arcas, M. Martínez de la Escalera y A. Cobos dejaron en algunos individuos de la colección del Museo Nacional de Ciencias Naturales. Estas poblaciones sudorientales presentan una serie de rasgos morfológicos característicos que las hacen merecedoras de un estudio taxonómico independiente. Desafortunadamente, los caracteres diagnósticos de estas poblaciones no han sido puestos de manifiesto adecuadamente en las escasas publicaciones donde se menciona el taxón (Górriz, 1882), o incluso han sido interpretados poco apropiadamente, como en la clave de Rodríguez LópezNeyra (1914), en la que se incluye erróneamente a $M$. insignis entre las especies con el último artejo de las antenas simple. Esta falta de consistencia y la escasez de información han propiciado la inclusión de $M$. insignis en la sinonimia de $B$. majalis (Borchmann, 1917; Bologna, 1988; 1991).

En este trabajo presentamos un estudio de la morfología externa y de la distribución geográfica de Berberomeloe, en el que se ponen de manifiesto los caracteres diagnósticos de $B$. insignis frente a $B$. majalis. La constancia y relevancia de estos caracteres, unida a una distribución geográfica reducida, adyacente a la de $B$. majalis, permite atribuir un estatus específico a $B$. insignis, que por lo tanto constituye la segunda especie del hasta ahora monotípico género Berberomeloe (Bologna, 1988, 1991).

\section{Material y métodos}

El material estudiado incluye 376 ejemplares ibéricos y norteafricanos del género Berberomeloe, de las colecciones del Museo Nacional de Ciencias Naturales (CSIC) de Madrid, de la colección de la Universidad de Alicante y de la colección privada de A. J. Zuzarte (Portugal). Gran parte de los ejemplares examinados proceden de las colecciones históricas de L. Pérez Arcas, M. M. de la Escalera, C. Bolívar y otros conocidos entomológos españoles, y aunque alguno de estos ejemplares ya fue citado en obras previas (Fuente, 1933), hasta ahora no se había efectuado una revisión crítica de los mismos.

El material examinado cubre la mayor parte de la distribución geográfica del género (Fig. 1), con excepción de Túnez y el sur de Francia, áreas geográficas que han sido estudiadas adecuadamente por Bologna (1988; 1991). La lista completa del material examinado se presenta en el Apéndice I.

El análisis de la morfología externa y las ilustraciones se efectuaron utilizando material prepara- 


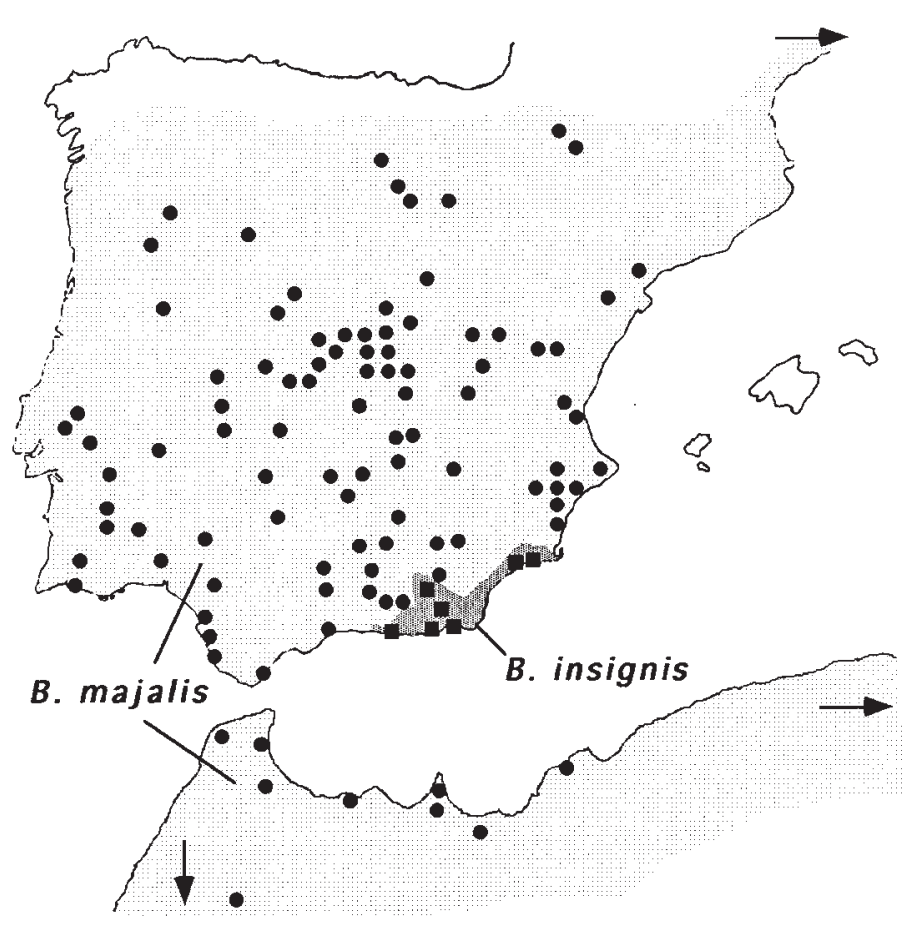

Fig. 1.- Mapa de la Península Ibérica y del extremo noroeste de Africa con la distribución geográfica de Berberomeloe majalis (sombreado claro) y $B$. insignis (sombreado oscuro). Los círculos señalan la procedencia del material examinado de $B$. majalis, los cuadrados de $B$. insignis. Las flechas indican áreas hacia donde se extiende la distribución de $B$. majalis (ver texto).

Fig. 1.- Map of the Iberian Peninsula and Northwestern Africa showing the geographic range of Berberomeloe majalis (light shade) and B. insignis (dark shade). Localities indicating the procedence of material examined are represented by circles for $B$. majalis and squares for $B$. insignis. Arrows point to the regions where $B$. majalis is also present (see text).

do en seco, las genitalias se prepararon a partir de ejemplares secos o conservados en alcohol de $70^{\circ}$.

\section{Resultados}

Los Lyttini del género Berberomeloe son fácilmente reconocibles por su aspecto voluminoso, con el abdomen muy engrosado y los élitros reducidos, ápteros, con las antenas relativamente largas y el pronoto sin salientes tuberculares laterales. Bologna $(1988$; 1991) proporciona una diagnosis detallada del género. Sus larvas de primera edad son de tipo marchador, no foréticas, lo que permite separarlos indiscutiblemente de las especies de la tribu Meloini con las que comparte un aspecto externo similar (Górriz, 1878; 1882; Cros, 1912; Bologna, 1988). El género tiene una distribución mediterráneooccidental, las larvas son parásitas de abejas solitarias (Apoidea), los adultos suelen localizarse en terrenos secos y con escasa vegetación arbórea, y a menudo se encuentran deambulando por caminos y carreteras (Valladares, 1984; Bologna, 1988).

Los ejemplares estudiados del género Berberomeloe se pueden agrupar en dos unidades claramente diferenciables y relativamente homogéneas (Fig. 1). Un grupo, que responde a la diagnosis de $B$. insignis, comprende únicamente las poblaciones procedentes de la franja costera de Granada, Almería y Murcia; el otro grupo, atribuible a B. majalis, incluye a todas las poblaciones restantes estudiadas. En los siguientes parráfos se describen los caracteres diagnósticos y la variabilidad de cada uno de estos grupos.

\section{Berberomeloe insignis (Charpentier, 1818) stat. nov.}

Meloe insignis Charpentier, 1818: 258, lám. 3, fig. 1. Meloe majalis var. insignis Charpentier: Escherich, 1896: 29.

Berberomeloe majalis: Bologna, 1988: 361 (pars). Bologna, 1991: 105 (pars).

Diagnosis. Especie de gran tamaño caracterizada por los siguientes caracteres (Figs. 2 y 3): (1) sienes muy desarrolladas y salientes; (2) antenas largas con los segmentos IV al X alargados y dentados, especialmente los segmentos V, VII y IX del macho que poseen un diente externo muy desarrollado; (3) ángulos anteriores del pronoto salientes; (4) pronoto casi tan largo como ancho, con los lados divergentes hacia delante; (5) puntuación cefálica y pronotal casi ausente, con punteaduras gruesas poco profundas escasas y dispersas; (6) ápice de los parámeros estrecho y alargado; y (6) coloración particular, con las sienes de color rojoanaranjado y la parte posterior de los terguitos abdominales de color negro.

DESCRIPCIÓN. La descripción original de Charpentier (1818) es muy breve y se centra en la coloración. Los autores que mencionan posteriormente a este taxón (Górriz, 1882; López-Neyra, 


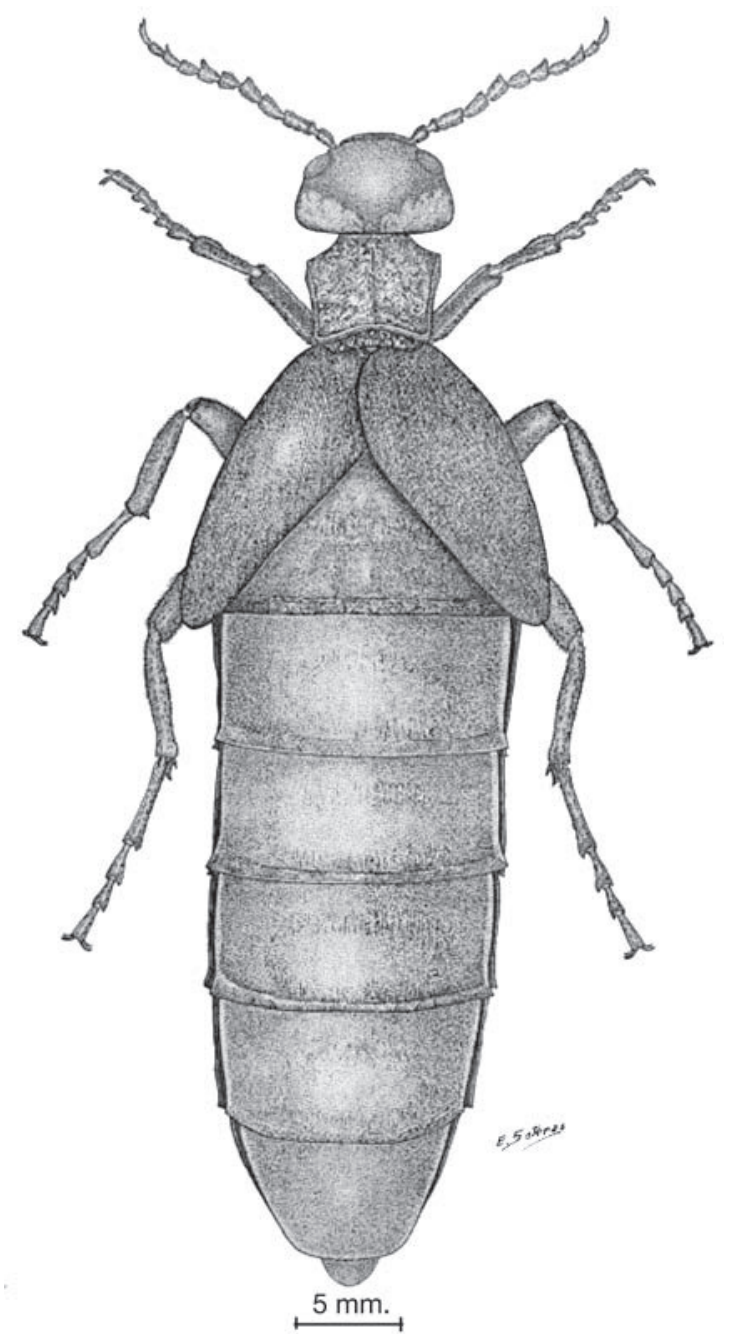

Fig. 2.- Habitus de un macho de Berberomeloe insignis de Motril (Granada). Nótese la forma de los artejos antenales y del pronoto y el patrón de coloración cefálica.

Fig. 2.- Habitus of a male of Berberomeloe insignis from Motril (Granada). The shape of the antennal segments, of the pronotum and the coloration of the head are distinctive.

1914) no ampliaron esta descripción por falta de material, por lo aquí se redescribe siguiendo el patrón utilizado por Bologna (1988) para B. majalis: Longitud del cuerpo muy variable, de 12 a 49 $\mathrm{mm}$, aunque la mayor parte de los ejemplares examinados tienen tallas más próximas al límite superior. Cuerpo de color negro, subopaco, con la cabeza generalmente más brillante. Cabeza ancha, ligeramente convexa, más ancha que el pronoto; sienes muy desarrolladas, particularmente en los ejemplares de mayor tamaño, de forma que la cabeza toma un aspecto trapezoidal (Figs. 3d y 4), las sienes son de color rojo o anaranjado y esta coloración se extiende en algunos individuos hasta el borde posterior del ojo; la frente es subconvexa, sin puntos rojos o con ellos, a veces con una mancha frontal rojo-anaranjada extendida en forma de $\mathrm{V}$ o de $\mathrm{Y}$ (Fig. 4); los ojos apenas sobresalen; la cabeza posee punteaduras dispersas desiguales $(0.039-0.070 \mathrm{~mm}$ de diámetro, separadas entre sí de 0.163 a 1.022 $\mathrm{mm}$ ), es glabra excepto en las proximidades de la base de las antenas; la sutura frontal es curva; el clípeo es transverso y tiene punteaduras gruesas dispersas, con pelos gruesos en las porciones laterales; el labro tiene punteaduras setosas dispersas, es transverso y tiene el borde anterior casi recto; mandíbulas robustas, el segmento terminal de los palpos maxilares algo dilatado apicalmente. Antenas con 11 segmentos, alargadas, más largas y más comprimidas en los machos, las antenas sobrepasan la base del pronoto en los machos; en los machos (Fig. 3f) el artejo I está dilatado apicalmente y completamente cubierto por pilosidad gruesa y larga; el artejo II es muy corto y también cubierto de pelos gruesos; el segmento III es aproximadamante 5 veces más largo que el II, es subcilíndrico y está ligeramente dilatado hacia el ápice; el artejo IV es más corto que el III y está, al igual que los segmentos $\mathrm{V}$ al X, dentado apicalmente; el desarrollo de los dientes apicales externos es máximo en los segmentos V, VII y IX, mientras que los artejos VI, VIII y IX tienen un aspecto más alargado y los dientes están menos desarrollados; en algunos ejemplares, el ápice de los dientes externos es de color amarillo claro; el artejo XI es alargado, presenta un depresión logitudinal corta y un diente anterior corto, por lo que toma un aspecto bifurcado; en las hembras los segmentos antenales IV al X son más cortos, subrectangulares y tienen los dientes menos marcados; el diente del artejo XI casi no se marca. El protorax es subcuadrangular (Fig. 3e), casi tan largo como ancho; los lados del pronoto están ligeramente levantados y divergen hacia la parte anterior, el margen posterior está finamente rebordeado y está ligeramente arqueado; los ángulos anteriores están muy marcados y sobresalen lateralmente, de forma que confieren al pronoto un aspecto casi pentagonal; la superficie pronotal es lisa con una línea media poco profunda marcada en los dos tercios posteriores, y con pequeñas arrugas poco definidas y punteaduras gruesas superficiales muy dispersas desiguales $(0.126-0.168 \mathrm{~mm}$ de diá- 


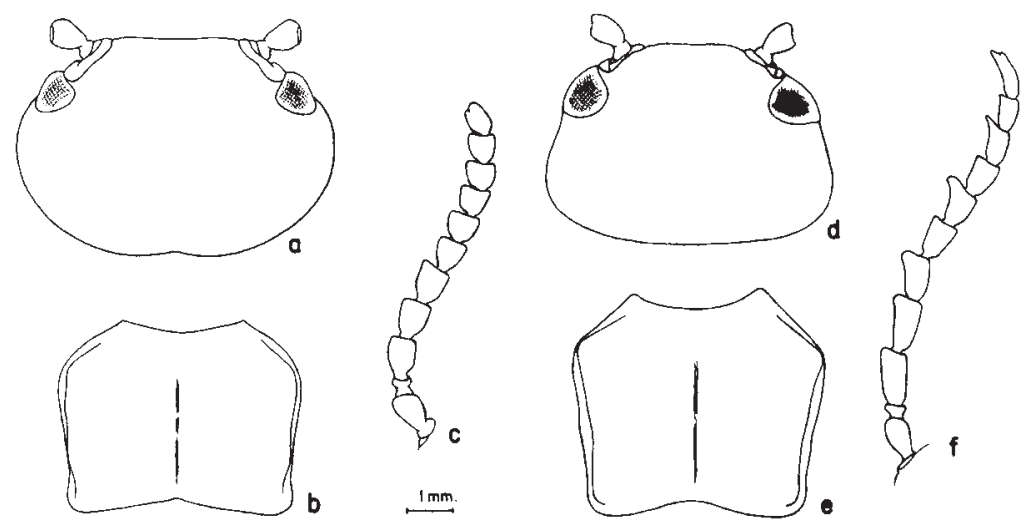

Fig. 3.- Detalles de la morfología externa de Berberomeloe. B. majalis (Madrid): 3a: cabeza; 3b: pronoto; 3c: antena. B. insignis (El Alquián, Almería) : 3d: cabeza; 3e: pronoto; $3 \mathrm{f}$ : antena.

Fig. 3.- Aspects of the external morphology of Berberomeloe. B. majalis (Madrid): 3a: head; 3b: pronotum; 3c: antenna. B. insignis (El Alquián, Almería): 3d: head; 3e: pronotum; 3f: antenna.

metro, separadas entre sí de 0.120 a $0.990 \mathrm{~mm}$ ), más concentradas hacia la línea media. Mesonoto y metanoto no visibles dorsalmente. Prosterno estrecho, arqueado anteriormente. Mesosterno ensanchado lateralmente, con el área marginal estrecha y la parte posterior levantada y definida por una depresión muy marcada. Metasterno corto y estrecho posteriormente. Patas relativamente robustas. Tibias cubiertas de pelos gruesos y cortos, menos densos en las posteriores; poseen dos espolones terminales en cada tibia, los de las anteriores y medias son subiguales, delgados y puntiagudos, los de las posteriores son muy desiguales, el interno es más grueso y está dilatado casi espatulado terminalmente. Las coxas medias se solapan parcialmente con las posteriores. Las uñas son lisas. Élitros más cortos que el abdomen, imbricados en la base, dehiscentes, que únicamente cubren completamente el primer terguito abdominal, y parte del segundo; la depresión humeral es corta y superficial. Abdomen muy voluminoso. Terguitos y esternitos con puntuación densa, de forma que poseen un aspecto mate; la porción posterior de los terguitos es rugo$\mathrm{sa}$; los márgenes posteriores de cada terguito están poco esclerotizados y son de color totalmente negro. Margen apical del esternito VII, arqueado en el macho, algo menos en la hembra; último esternito visible del macho ampliamente emarginado, y redondeado en la hembra. Parámeros en vista lateral con el cono apical tan largo como la base. Pene con harpagae de tamaño similar separados entre sí y gancho terminal muy arqueado. (Fig. 5).

VARIABILIDAD. La talla es muy variable, pero las diferencias más llamativas no corresponden a la longitud total si no al desarrollo relativo de la cabeza; en ejemplares de 45 a $48 \mathrm{~mm}$ de longitud la anchura de la cabeza varía de 5 a $8 \mathrm{~mm}$. La forma de la cabeza es variable y en algunos individuos las sienes están poco desarrolladas, como en $B$. majalis, aunque en otros su desarrollo modifica completamente el contorno de aquella que toma un aspecto subtriangular. El brillo de la cabeza es variable, y a veces es completamente mate. Los dientes externos de los segmentos antenales están marcados en todos los ejemplares, aunque las hembras muestran un desarrollo de los segmentos menor, similar al alcanzado habitualmente por los machos de $B$. majalis. La extensión de las manchas cefálicas, especialmente de la mancha frontal, es variable (Fig. 4). Un ejemplar de Cartagena y otro de Mazarrón (Murcia), son de color negro uniforme incluida la región cefálica. En algunos ejemplares los élitros pueden llegar a cubrir completamente el abdomen.

DisTRIBUCIÓN GEOGRÁFICA. Se trata de una especie endémica de una pequeña franja costera del sureste peninsular, con cortas penetraciones hacia el interior a lo largo del Valle de Guadix, y limitada a las provincias de Granada, Almería y Murcia (Fig. 1). Posiblemente gran parte de las localidades indicadas por Ruiz et al. (1994) para B. majalis, correspondan realmente a esta especie. Hasta el momento no se ha localizado en simpatría con $B$. majalis. La posible zona de contacto entre $B$. insignis y $B$. majalis podría situarse en zonas de media altitud, al pie de Sierra Nevada en Almería y Granada, y al sur de Sierra Espuña en Murcia, y se desconoce la posible extensión y naturaleza de la misma. La identificación de ambas especies en las zonas de contacto puede verse dificultada debido a que las poblaciones sudoccidentales de $B$. majalis presentan una coloración negra uniforme, sin las características bandas transversales rojas, y la pun- 

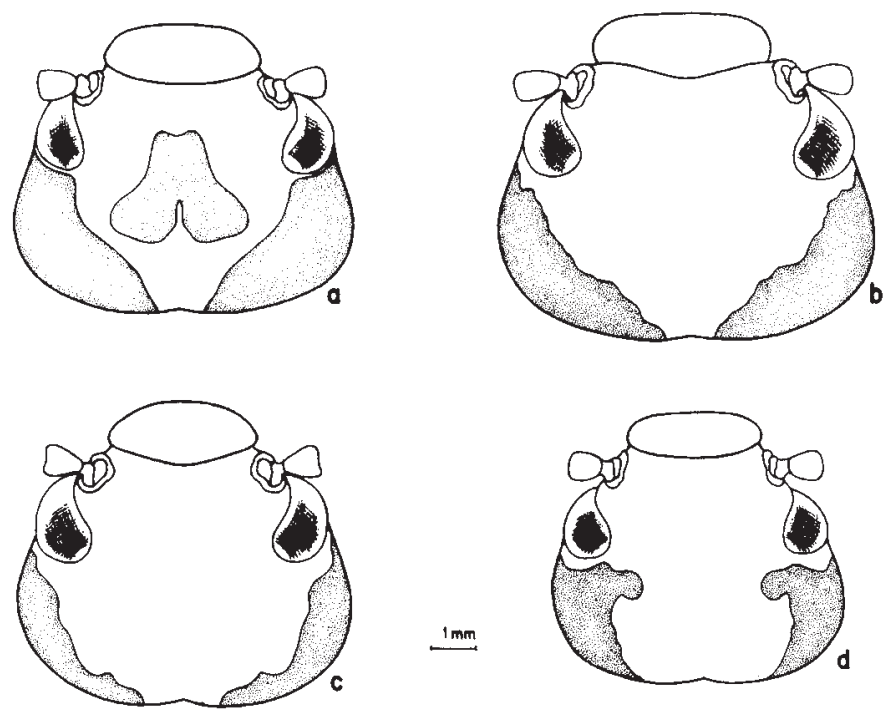

Fig. 4.- Variabilidad de la forma de la cabeza y de la extensión de las manchas rojas cefálicas en cuatro individuos de B. insignis. 4a: Gérgal (Almería); 4b: El Alquián (Almería); 4c: Moreda (Granada); Motril (Granada).

Fig. 4.- Relative extension of the cephalic red marks and variability of head shape in four specimens of B. insignis. 4a: Gérgal (Almería); 4b: El Alquián (Almería); 4c: Moreda (Granada); Motril (Granada).

tuación del pronoto y cabeza dispersa, similar a la de $B$. insignis, bien diferente de la puntuación gruesa y confluente que presentan los ejemplares de $B$. majalis de la Meseta. Sin embargo la morfología antenal y la forma de la cabeza y pronoto, permiten identificar con facilidad a los ejemplares de esta región.

ECología. Los únicos datos fenológicos disponibles indican la presencia de adultos a finales del invierno y primavera, desde diciembre hasta abril. La distribución de la especie parece estar restringida a los pisos bioclimáticos termo y mesomediterráneos, sin que existan otros datos ecológicos disponibles.

Comentarios taxonómicos. Desde que Charpentier (1818) describió la especie muy pocos autores han tenido oportunidad de observar ejemplares de la misma, por lo que gran parte de los cambios taxonómicos que ha sufrido se han basado únicamente en la opinión de autores anteriores. Reitter (1895) incluye el nombre de $M$. insignis entre diversas variedades de $M$. majalis en una nota a pie de página de sus claves, pero no especifica si la considera como tal, ya que el nombre que señala es el específico. Además precisa que no ha visto material de M. insignis. Escherich (1896), sin embargo, la considera una variedad de $M$. majalis haciendo referencia a la obra de Reitter (op. cit.), y a partir de ese momento todos los autores posteriores siguen su opinión (Borchmann, 1917; Fuente, 1933), hasta que Bologna (1988) la incluye en la sinonimia de $B$. majalis. Charpentier (1818) presenta una figura de la especie en la que se pone de manifiesto la coloración cefálica particular por lo que no se ha considerado necesario revisar el tipo de la especie.

Berberomeloe majalis (Linnaeus, 1758)

Meloe majalis Linnaeus, 1758: 419.

Meloe laevigatus Olivier, 1795: 6, lám. 1, fig. 5a, b. Meloe maculifrons Lucas, [1846]: 399, lám. 33, fig. 3.

Meloe majalis var. maculicollis Reitter, 1872: 176.

Meloe frontalis Dejean i.1. (Baudi, 1878: 355).

Meloe fissicornis Górriz, 1882: 38.

Meloe majalis var. sanguineus Escherich, 1889: 334 (nom. nudum).

Trichomeloe majalis (Linnaeus): MacSwain, 1956: 71.

Berberomeloe majalis (Linnaeus): Bologna, 1988: 361.

DiAGNOSIS. Especie de talla muy variable caracterizada por los siguientes rasgos (Fig. 3): (1) sienes poco desarrolladas y apenas o nada salientes; (2) antenas cortas con los segmentos IV al X subtrapezoidales y dentados, pero sin dientes externos muy desarrollados; (3) ángulos anteriores del pronoto poco o nada salientes; (4) pronoto subcuadrangular, con los lados paralelos; (5) puntuación cefálica y pronotal gruesa, en muchas ocasiones confluyente; (6) ápice de los parámeros estrecho y corto; y (6) coloración particular, con las sienes de color negro y la parte posterior de los terguitos abdominales generalmente de color rojo o anaranjado.

DESCRIPCIÓN. La descripción de esta especie realizada por Bologna $(1988$; 1991) es muy completa y a ella nos remitimos. La genitalia del macho ha sido figurada por Valladares $(1984: 133) \mathrm{y}$ Bologna (1988: 361; 1991: 105).

VARIABILIDAD. Se trata de una especie relativamente variable con tendencias geográficas defini- 

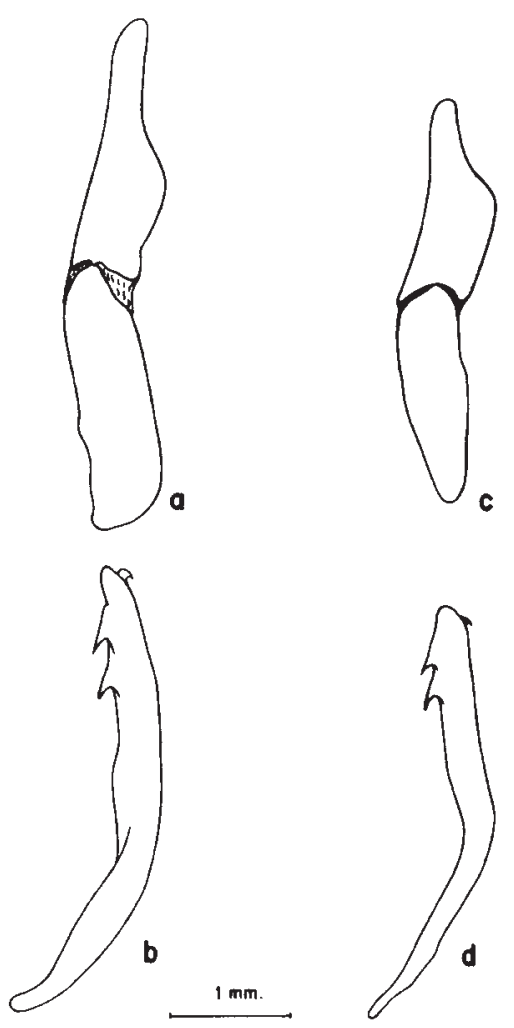

Fig. 5.- Aspectos de la genitalia del macho de Berberomeloe. 5a: parámeros, y 5b: pene con los harpagae de B. insignis (Motril, Granada). 5c y 5d: parámeros y pene de B. majalis (Madrid).

Fig. 5.- Male genitalia of Berberoneloe. 5a: parameres, and 5b: aedeagus with harpagae of $B$. insignis (Motril, Granada). $5 \mathrm{c}$ and $5 \mathrm{~d}$ : parameres and aedeagus of $B$. majalis (Madrid).

das. Las poblaciones del norte de Africa son extremadamente variables en la puntuación de la cabeza $\mathrm{y}$ del pronoto; todos los ejemplares que hemos examinado de Marruecos y Argelia poseen las bandas abdominales anaranjado-rojizas características de la especie. Dentro de la Península Ibérica pueden distinguirse dos grupos de poblaciones muy similares en aspecto general pero bien definidos por la puntuación de la cabeza y del pronoto. Los ejemplares de las poblaciones localizadas al sur del Guadalquivir y en las porciones sudorientales de la Península (en las provincias de Cádiz, Málaga, Granada, parte de Jaén, Murcia y parte de Alicante), presentan el pronoto y la cabeza apenas punteados, casi lisos, por lo que éstos generalmen- te toman un aspecto brillante. Por el contrario las poblaciones de la Meseta y del norte y del oeste peninsular, con independencia de la presencia o ausencia de bandas abdominales anaranjadas, se caracterizan por presentar el pronoto y cabeza fuertemente punteados con fosetas de 0.039 a 0.143 $\mathrm{mm}$ de diámetro (separación máxima entre puntos $0.232 \mathrm{~mm}$ ). Estos puntos a menudo confluyen entre sí, sobre todo en el pronoto que generalmente toma un aspecto mate. En las poblaciones del centro y sur de Portugal los puntos tienden a estar más dispersos, pero siempre están mucho más marcados y en mayor densidad que en las poblaciones béticas. Los rasgos de las poblaciones béticas son aparentemente constantes en la mayor parte de su área de su distribución por lo que ambos grupos de poblaciones (bético y centro-occidental) posiblemente deberían ser tratados como subespecies. Sin embargo, antes de tomar una decisión taxonómica concerniente al estatus de estas dos razas geográficas se hace necesario un estudio detallado de su variabilidad, especialmente de las zonas potenciales de contacto, en las estribaciones de Sierra Morena, las Sierras Béticas y las regiones noroccidentales de Málaga y Cádiz.

La coloración predominante de las poblaciones noroccidentales es negra con una banda transversal rojo-anaranjada dorsal en la parte posterior de cada segmento abdominal. En los yesos de Madrid (Paracuellos, Montarco, Rivas, Vicálvaro, San Fernando) y en el Algarve portugués todos los ejemplares examinados son de coloración uniformemente negra. En zonas silíceas del sur del Sistema Central conviven ambos morfotipos de coloración (Paterna del Campo, El Pardo, Arenas, Cobeña). Dentro del grupo bético, todos los individuos examinados de las Sierras Penibéticas: Sierra Nevada, Sierra Carbonera, Güéjar Sierra presentan una coloración completamente negra, mientras que en zonas de menor altitud de Cádiz y Málaga la coloración dominante es negra con las bandas rojizas transversales. La longitud de las bandas rojoanaranjadas es variable; en los individuos del oeste peninsular suele limitarse a la zona central del borde posterior del terguito, mientras que en el este y noreste la banda de color suele extenderse por casi todo el margen posterior, tomando, en vista dorsal, la apariencia de un anillo. La coloración en vivo de esta banda post-tergal varía desde el rojo fuerte, predominante en zonas calcáreas, al amarillo anaranjado que domina en los terrenos graníticos, aunque estas variaciones también pueden presentarse a nivel local. Algunos ejemplares presen- 
tan una mancha rojiza frontal (Pinos Puente, Baeza, Saceruela). La longitud de los ejemplares que hemos estudiado es muy variable (de 8 a $51 \mathrm{~mm}$ ) y pueden alcanzar hasta $75 \mathrm{~mm}$ (Bologna, 1988). Entre los ejemplares de menor talla suelen incluirse individuos con los élitros más largos que el abdomen. Estos individuos de abdomen reducido se han observado en Baeza, Madrid, Castillo del Val, El Vado, Cuenca y Valsequillo, estos últimos extraordinariamente diminutos.

DistRIBUCIÓN GEOGRÁFICA. Especie extendida por el noroeste de Africa, la Península Ibérica y una estrecha franja del sur de Francia (Bologna, 1991). De acuerdo con la procedencia de los ejemplares examinados es una especie presente en toda la región mediterránea peninsular, alcanzando por el norte las estribaciones de la Cordillera Cantábrica, el País Vasco y las cadenas Prepirenaicas de Huesca (Fig. 1). Ha sido citada además en numerosas localidades ibéricas (Dieck, 1870; Salvañá, 1870; Martínez y Sáez, 1873; Uhagón, 1887; Cuní y Martorell, 1888; Medina, 1895; Górriz, 1902; Redondo, 1915; Fuente, 1933; Soumakov, 1934; Cobos, 1949; Valladares y Salgado, 1983; Bologna, 1988).

ECOLOGía. Las fechas de captura de los individuos se extienden predominantemente durante abril y mayo en zonas de media o baja altitud y durante junio y julio en zonas de montaña, datos que coinciden con las observaciones de Valladares y Salgado (1983) y Bologna (1988). Alcanza cerca de los $3000 \mathrm{~m}$ en Sierra Nevada (Veleta, Loma del Telégrafo, Mulhacén) (Bologna, 1988; 1991 у datos propios) aunque en el resto peninsular suele ocupar cotas altitudinales inferiores a los $1500 \mathrm{~m}$.

\section{Discusión}

El examen del material de la colección del MNCN pone de relieve que todas las Berberomeloe de una estrecha franja costera del sureste ibérico se caracterizan por rasgos morfológicos constantes que responden a la diagnosis de $B$. insignis. Entre estos caracteres diferenciales destacan: una morfología cefálica característica que incluye un desarro1 lo amplio de las sienes; antenas largas con los segmentos IV al X alargados y dentados, especialmente los segementos V, VII y IX de los machos, que poseen un diente externo muy desarrollado; ángulos anteriores del pronoto pronunciados; pronoto con los lados divergentes anteriormente; puntuación cefálica y pronotal escasa y dispersa; edeago con el cono apical de los parámeros muy largo; y finalmente por una coloración particular, con las sienes de color rojo-anaranjado y la parte posterior de los terguitos abdominales de color negro. Estos caracteres, exceptuando la puntuación cefálica y pronotal, no aparecen en ninguna otra población de Berberomeloe.

Estas diferencias morfológicas y la definición de los límites de su distribución geográfica son, a nuestro juicio, razones suficientes para que estos grupos sean tratados como especies independientes. El área geográfica ocupada por $B$. isignis es tan reducida que, en el caso de no existir barreras reproductivas que aislen estos dos taxones geográficamente adyacentes y sin barreras geográficas que los separen, la simple dispersión de $B$. majalis en el área de $B$. insignis provocaría la extinción rápida de ésta vía hibridación o daría lugar a la formación de una mezcla más o menos homogénea de fenotipos que no correspondería a una estructura geográfica definida como la que se observa actualmente. Por lo tanto consideramos que $B$. insignis debe ser considerada a nivel específico. El género Berberomeloe queda constituido por dos especies bien definidas, que pueden separarse según la clave siguiente:

1.- Cabeza con las sienes prominentes, rojo-anaranjadas y a menudo con una mancha frontal en Y. Puntuación cefálica casi ausente. Machos con los artejos antenales alargados con un diente muy desarrollado en los artejos V, VII y IX. Pronoto con los ángulos anteriores muy salientes, y lados convergentes posteriormente. Coloración general negra excepto en la región cefálica ............................ B. insignis

* Cabeza con las sienes redondeadas, de color totalmente negro. Puntuación cefálica generalmente muy densa o espaciada pero siempre marcada. Si existe una mancha frontal rojiza es redondeada. Machos con los artejos antenales trapezoidales y con dientes externos poco marcados. Pronoto con los ángulos anteriores rectos, apenas salientes y lados paralelos. Coloración general variable, negra, o con más frecuencia con una banda transversal roja o anaranjada en la parte posterior de cada terguito abdominal

B. majalis

La distribución geográfica de $B$. insignis resulta muy peculiar, ya que la mayoría de los Lyttini de zonas templadas tienen distribuciones geográficas relativamente amplias. Además, los factores históricos que han podido conducir a su aislamiento resultan poco evidentes. Aunque $B$. insignis es un endemismo de una zona geográfica muy reducida y posiblemente sea el meloideo ibérico con una dis- 
tribución más restringida, no podemos argüir razones de tipo ecológico que justifiquen su aislamiento, ya que coloniza tanto las zonas áridas termomediterráneas costeras, como las faldas de las Sierras de Gádor y Filabres, sometidas a un clima y poseedoras de una vegetación completamente distinta de la costera. El área que ocupa $B$. insignis es rica en endemismos vegetales e incluso en insectos (Cobos, 1987) pero la mayor parte de ellos tienen unos requerimientos ecológicos mucho más específicos de los que parece necesitar $B$. insignis. Quizás el conocimiento de sus hospedadores larvarios, hasta ahora desconocidos, pueda aportar nueva luz sobre este problema.

Se han descrito eventos paleogeográficos que podrían haber causado el aislamiento de taxones con poca capacidad de dispersión en la zona bética. Entre estos eventos vicariantes cabría destacar la formación del Estrecho de Gibraltar que separó definitivamente las poblaciones europeas y africanas en el límite Mioceno-Plioceno (Busack, 1986), o la formación de las cuencas fluviales ibéricas, en particular la del Guadalquivir, que mantuvo separadas las poblaciones nor- y sub-béticas durante el Plioceno (ver por ejemplo Arntzen y García-París, 1995; García-París et al., 1998). Sin embargo, la distribución actual de $B$. majalis, que a pesar de ser una especie no forética con limitada capacidad de dispersión, ocupa ambos lados del Estrecho de Gibraltar, y también ambas orillas de la Cuenca del Guadalquivir, resulta muy difícil de justificar basándose en esos escenarios paleogeográficos, a no ser que se invoquen procesos de dispersión relativamente recientes. La puntuación cefálica y protorácica de las poblaciones sub-béticas de $B$. majalis se aproxima mucho más a la de $B$. insignis que a la de las poblaciones nor-béticas, y podría ser indicativo de un contacto antiguo o de cierto grado de intercambio genético entre ellas, lo que apoyaría la hipótesis paleogeográfica, pero también podría ser el resultado de convergencia, o paralelismo, o simplemente tratarse de un carácter primitivo retenido por ambos grupos de poblaciones. Por lo tanto, mientras no dispongamos de otros caracteres, en especial de tipo molecular, o de otro tipo de evidencia biológica, cualquiera de estas hipótesis sobre la evolución de $B$. insignis es especulativa.

No deja de resultar sorprendente que una especie llamativa, de más de $4 \mathrm{~cm}$ de longitud, y con un aspecto externo inconfundible, haya pasado desapercibida en la literatura científica. Quizás, para los investigadores no ibéricos, la falta de material disponible condujo a que haya sido considerada como una simple variación individual, mientras que, para los ibéricos, que posiblemente nunca dudaron de su validez (y etiquetaron en consecuencia el material de sus colecciones), resultaba una cuestión demasiado evidente para merecer un estudio detallado. Sea como fuere, el reconocimiento de B. insignis, uno de los endemismos más llamativos y restringidos del sureste español, plantea numerosas cuestiones biológicas y evolutivas que ponen de manifiesto su interés científico. Además no podemos olvidar que su área de distribución se localiza en una de las zonas españolas más afectadas por el urbanismo turístico y por los cultivos intensivos, y que por lo tanto, es necesario un estudio de su estado de conservación.

\section{Agradecimientos}

Agradezco a Emilio Soteras los dibujos de Berberomeloe que completan este trabajo. A Carolina Martín y Mercedes París su ayuda en la consulta y revisión de la colección del Museo Nacional de Ciencias Naturales de Madrid. A Eduardo Galante el facilitarnos la revisión de la colección de meloideos de la Universidad de Alicante. A Antonio José Zuzarte por poner a nuestra disposición su colección particular de meloideos de Portugal. A Elena Pérez Zapirain, a Mercedes París, a F. Ángel Montes y a Esther Izquierdo los ejemplares que nos han proporcionado. Al proyecto PB97-1231 y al Departamento de Colecciones del MNCN la ayuda prestada. Durante el periodo de realización de este trabajo MG-P ha disfrutado una beca postdoctoral de la Comunidad de Madrid.

\section{Referencias}

Arntzen, J. W. \& García-París, M., 1995. Morphological and allozyme studies of midwife toads (genus Alytes), including the description of two new taxa from Spain. Cont. Zool., 65: 5-34.

BAudi di Selve, F., 1878. Heteromera in catalogo Dejeani. Pars sexta. Dt. ent. Z., 22: 337-376.

Bologna, M. A., 1988. Berberomeloe, a new west Mediterranean genus of Lyttini for Meloe majalis Linné (Coleoptera, Meloidae). Systematics and bionomics. Boll. Zool., 55: 359-366.

Bologna, M. A. 1991. Fauna de Italia. Coleoptera Meloidae. Calderini. Bologna. 541 pp.

Borchmann, F., 1917. Pars 69. Meloidae, Cephaloidae. En: Schenkling, E. (ed.) Coleopterorum Catalogus auspiciis et auxilio W. Junk. Junk, Berlin. 208 pp.

BusACK, S. D., 1986. Biogeographic analysis of the herpetofauna separated by the formation of the Strait of Gibraltar. Natl. Geogr. Res., 2:17-36.

Charpentier, T. DE, 1818. Meloe insignis, p. 258, en: Germar, E. F.. Vermischte Bemerkungen über einige Käferarten. Germ. Mag. Ent., 3: 228-260. 
Cobos, A., 1949. Datos para el catálogo de los coleópteros de España. Especies de los alrededores de Málaga. Bol. R. Soc. esp. Hist. nat., 47: 563-609.

CoBos, A., 1987. La coleopterofauna endémica almeriense. Graellsia, 43:3-17.

Cros, A., 1912. Moeurs et evolution du Meloe majalis L. Bull. Soc. Hist. nat. Afr. Nord, 4: 45-48, 70-72, 9396, 154-161, 181-190, 209-215.

Cros, A., 1913. Contribution à la biologie des Méloides algériens. Bull. Soc. Hist. nat. Afr. Nord, 5: 16-26.

Cros, A., 1928. Essai sur la forme contractée (hypnothèque ou pseudonymphe) des larves des Meloidae. Ann. Soc. ent. France, 17: 27-58.

Cros, A., 1940. Essai de classification des Meloidae algériens. VI Congr. Intern. Ent. Madrid, 1935: 312-338.

Cuní y Martorell, M., 1888. Insectos observados en los alrededores de Barcelona. An. Soc. esp. Hist. nat., 17: 133-191.

DIECK, G., 1870. Eine entomologische Wintercampagne in Spanien. Berl. ent. Z., 14: 145-184.

EsCHERICH, K., 1889. Nachträge und Berichtigungen zum Catalogus Coleopterorum von Gemminger und Harold, betreffend die gattung Meloë. Dt. ent. Z., 1889: 333-335.

EsCHERICH, K., 1896. Meloiden-studien. IV. Theil. Wien. ent. Ztg., 15:27-30.

Fuente, J. M. DE LA, 1933. Catálogo sistemático geográfico de los Coleópteros observados en la Península Ibérica, Pirineos propiamente dichos y Baleares. Bol. Soc. ent. Esp., 16: 18-49.

García-París, M.; Alcobendas, M. \& Alberch, P., 1998. Influence of the Guadalquivir River Basin on the evolution of Salamandra salamandra (Caudata: Salamandridae) from Southern Spain. Copeia, 1998: 173-176.

GóRriz, R. J., 1878. (Nota sobre meloideos). Actas Soc. esp. Hist. nat., 7: 71-73.

GóRRIZ, R. J., 1881. Nuevas observaciones sobre costumbres y metamorfosis de algunos vesicantes. Actas Soc. esp. Hist. nat., 10: 55-64.

GóRRIZ, R. J., 1882. Ensayo para la Monografía de los coleópteros Meloideos indígenas con aplicación a las ciencias médicas. Sanz y Navarro. Zaragoza. 201 pp.

GóRriz, R. J., 1902. Coleópteros de la Cuenca del Ebro. Bol. Soc. aragonesa Cienc. nat., 1: 21-23, 51-52, 154-155, 180-186.

Kaszab, Z., 1969. The system of the Meloidae (Coleoptera). Mem. Soc. entomol. ital., 48: 241-248.

LinNAEus, C., 1758. Systema Naturae per Regna tria Naturae, secondum Classes, ordines, genera, species, cum characteribus, differentiis, synonymis, locis. Ed. X, 1. Salvi. Holmiae. 824 pp.

LuCAS, P., [1846] Coléptères, en: Lucas, P. 1849. Exploration scientifique de l'Algérie pendant les années 1840, 1841, 1842, publiée par ordre du Gouvernement et avec le concours d'une commission. Historie Naturelle des Animaux Articulés. II. Coléoptères. Imprimerie Nationale. Paris. 590 pp. 47 tab.

MacSwaIn, J.M., 1956. A classification of the first instar larvae of the Meloidae (Coleoptera). Univ. Calif. Publ. Entomol., 12: 1-181.

MARTínez y SÁEZ, F. P., 1873. Datos sobre algunos coleópteros de los alrededores de Cuenca. An. Soc. esp. Hist. nat., 2: 53-75.

MedinA, M., 1895. Coleópteros de Andalucía existentes en el Museo de Historia Natural de la Universidad de Sevilla, clasificados por D. Francisco Martínez y Sáez. Actas Soc. esp. Hist. nat., 24: 25-61.

Mulsant, M. E., 1856. Histoire naturelle des Coléoptères de France. Vésicants. Magnin, Blanchard et Cie. París. 201 pp., 1 tab.

Olivier, A. G., 1792. Encyclopèdie mèthodique. Dictionnaire des Insectes, 7. Panckoucke. Paris. 827 pp.

Olivier, A. G., 1795. Entomologie, ou Histoire naturelle des Insectes. Coléoptères. Baudouin. Paris. 3. 557 pp.

PinTo, J. D. y SElander, R. B., 1970. The bionomics of the blister beetles of the genus Meloe and a classification of the New World species. Illin. Biol. Monogr., 42: 1-222.

PorTA, A., 1934. Fauna Coleopterorum italica. Vol. IV. Heteromera-Phytophaga. Piacentino. Piacenza. 415 pp.

Redondo, A., 1915. Coleopteros de Salamanca. Brotéria (Ser. Zool.) 1915: 14-48.

REITTER, E., 1872. Neue Käferarten von Oran gesammelt von Hans Leder. Berl. ent. Z., 16: 167-186.

REITTER, E., 1895. Bestimmungs-Tabellen der europaïschen Coleopteren Meloidae. 1. Theil: Meloini. 32. Ver. Verfassers, Paskau: 1-13.

Reitter, E., 1911. Fauna Germanica. Die Käfer des deutschen reiches. Nach der analytischen methode bearb. Lutz. Stuttgart. 3. 436 pp.

RodríGuEZ LóPEZ-NeYRA, C., 1914. Claves dicotómicas para la determinación de los meloideos indígenas. Bol. R. Soc. esp. Hist. nat., 14: 461-475.

Ruiz, J. L., SÁnchez-Piñero, F. y Avila, J. M., 1994. Faunística y corología de los Meloidae (Coleoptera) de zonas áridas de sureste de la Península Ibérica. Bolm. Soc. port. Ent., 2 (supl. 3) (Actas V Congr. Ibér. Entomol.): 325-335.

SalvaÑÁ, J. M., 1870. Apuntes para la geografía y fauna entomológicas de Mataró. Impr. Gregorio Juste. Madrid. $44 \mathrm{pp}$.

SuMAKOv, G., 1934. Inventa entomologica itineris hispanici et maroccani, quod a 1926 fecerunt Harald et Hakan Lindberg. XXI. Méloides du Bassin Méditerranéen et du Maroc. Comment. Biol. Soc. Sci. Fenn., 4(13): 1-6. 
Uhagón, S., 1887. Coleópteros de Badajoz. An. Soc. esp. Hist. nat., 16: 373-380.

VAlladares, L. F., 1984. Estudio de los coleópteros vesicantes de la provincia de León (Coleoptera, Meloidae). Institución "Fray Bernardino de Sahagún”, Diputación Prov. León, C.S.I.C. León. $156 \mathrm{pp}$.
Valladares, L. F. y Salgado, J. M., 1983. Contribución al conocimiento de los Meloidae (Col.) en la provincia de León. Bol. Asoc. esp. Ent., 7: 79-100.

Recibido, el 5-X-1998 Aceptado, el 26-XI-1998 Publicado, el 31-XII-1998

Apéndice I. - Material examinado. Las siglas MNCN corresponden a la coleccción del Museo Nacional de Ciencias Naturales de Madrid y UA a la colección de la Universidad de Alicante. Los nombres de las localidades se han escrito tal como aparecen en las etiquetas de los ejemplares, especialmente en los de Marruecos.

Berberomeloe insignis (Charpentier, 1818): EUROPA: ESPAÑA: ALMERIA: Gérgal: 1 ejemplar (MNCN). El Alquián: 24-IV-1942, 4 ejemplares (MNCN). El Palmer: 20-II1950, 2 ejemplares (A. Cobos Sánchez leg.) (MNCN). Rioja: 21-IV-1943, 1 ejemplar (M. Mendizabal leg.) (MNCN). Sabinal-Dalias: VII-1942, 1 ejemplar (MNCN). GRANADA: Moreda: 1 ejemplar (Uhagón leg.) (MNCN). Motril: 3 ejemplares (MNCN); 14-IV-1906, 1 ejemplar (Rivas Mateos leg.) (MNCN); III-1909, 2 ejemplares (Expedición del Museo) (MNCN). MURCIA: Cartagena: 4 ejemplares (Sánchez Gómez leg.) (MNCN). Mazarrón: XII-1924, 1 ejemplar (M. Escalera leg.) (MNCN).

Berberomeloe majalis (Linnaeus, 1758): ÁFRICA: ARGELIA: Orán: 4 ejemplares (MNCN). ESPAÑA: MELILLA: 1 ejemplar (MNCN); 1 ejemplar (R. Candel leg.) (MNCN). Melilla, Rostrogordo: 1 ejemplar (R. Candel leg.) (MNCN). MARRUECOS: Alhucemas, Beni Urriagel: VI-1932, 1 ejemplar (M. Escalera leg.) (MNCN). Azrou: 2 ejemplares (MNCN). Bab Taza, El Ajmes, Yebala: VI-1930, 1 ejemplar (Exp. C. Bolívar leg.) (MNCN). Mazagán: 1 ejemplar (MNCN). Montes Arrui: 1 ejemplar (A. Cabrera leg.) (MNCN). Sidi Salem, Kabila de Mazuca, 1 ejemplar (R. Candel leg.) (MNCN). Taurirt, 1 ejemplar (R. Candel leg.) (MNCN). Mequinéz: IV-1925, 1 ejemplar (M. Escalera leg.) (MNCN). Oujda: VII-1904, 1 ejemplar (M. Le Boul leg.) (MNCN). Tánger: 2 ejemplares (MNCN); 6 ejemplares (M. Escalera leg.) (MNCN); 1897, 2 ejemplares (MNCN); VI1905, 1 ejemplar (Escalera leg.) (MNCN). Tetuán: 1 ejemplar (MNCN). Timadit: 14-IV-1923, 3 ejemplares (MNCN). Tetuán a Ceuta: 1 ejemplar (MNCN). Segangan: 14-V-1902, 1 ejemplar (MNCN). Zoco el Arba de Ankeman, 1 ejemplar (R. Candel leg.) (MNCN).

EUROPA: ESPAÑA: Andalucía: 1 ejemplar (A. Kricheldorff leg.) (MNCN). ALBACETE: Bogarra, El Malojar: 8-III-1997, 1 ejemplar (M. París y E. Izquierdo leg.) (MNCN). Munera: 1 ejemplar (MNCN). Sierra del Agua: 11-V-1997, 1 ejemplar (E. Izquierdo leg.) (MNCN). ALICANTE: Alicante: 1 ejemplar (MNCN). 2 Km al E de Castell de Castells: 24-VI-1998, 1 ejemplar (M. García París y C. Martín leg.) (MNCN). Bosque Ilustrado, San Vicente del Raspeig: 31-III-1995, 1 ejemplar (B. Badia leg.) (UA). Campus Universidad: 13-V-1996, 1 ejemplar (UA). Chaparrrales, Petrel: 18-V-1996, 1 ejemplar (A. Vera leg.) (UA). Chorret de Catí, Petrel: 900 m, 18-V-1996, 11 h, 1 ejemplar (J. A. Serrano leg.) (UA). El Rebolledo: 10-V-1996, 1 ejemplar (P. Agulló leg.) (UA). Elche: 7-IV-1995, 1 ejemplar (J. Lozano leg.) (UA). Fontcalent: 13-IV-1997, 1 ejemplar (G.
Rodríguez leg.) (UA). Guardamar del Segura: 27-I-1997, 1 ejemplar (S. Lorenzo leg.) (UA); 9-III-1997, 1 ejemplar (G. Rodríguez leg.) (UA); 10-IV-1997, 1 ejemplar (P. de Andrés leg.) (UA). Ibi: 10-IV-1996, 1 ejemplar (Fabregat leg.) (UA). Los Campos, Ibi: 5-V-1997, 1 ejemplar (A. B. Talavera leg.) (UA). San Juan: 17-III-1996, 1 ejemplar (M. C. Poveda leg.) (UA); 25-III-1996, 1 ejemplar (P. Martínez leg.) (UA). San Vicente: 1-IV-1993, 1 ejemplar (J. D. Ordoñez leg.) (UA); 30III-1995, 1 ejemplar (T. Tweroy leg.) (UA); 12-II-1997, 1 ejemplar (B. Gisbert leg.) (UA). Sax: 14-IV-1996, 1 ejemplar (M. Gil Saucedo leg.) (UA). Sierra de Onil: 3-V-1994, 1 ejemplar (M. G. Cartagena leg.) (UA). Torremanzanas: 11-V-1995, 1 ejemplar (M. Hita leg.) (UA). Vall d'Ebo: 4-IV-1995, 1 ejemplar (N. Marcos leg.) (UA). Villena: 4-V-1997, 1 ejemplar (J. Tornero leg.) (UA). ÁVILA: Arenas: 1 ejemplar (J. Ardois leg.) (MNCN). Ávila: 1 ejemplar (J. Sanz leg.) (MNCN). Monbeltrán: 4 ejemplares (J. Ardois leg.) (MNCN). La Cañada: 1 ejemplar (C. Rodríguez leg.) (MNCN). $10 \mathrm{Km}$ al S de Las Navas del Marqués: 10-V-1998, 7 ejemplares (M. García París y C. Martín leg.) (MNCN). Poyales: 1 ejemplar (J. Ardois leg.) (MNCN). Sierra de Guisando: 1 ejemplar (MNCN). BADAJOZ: Jerez de los Caballeros-Fregenal de la Sierra: 2-V-1997, 1 ejemplar (J. París y M. García Rollán leg.) (MNCN). Magacela: 1 ejemplar (Boscá leg.) (MNCN). BURGOS: Castillo del Val: 1 ejemplar (J. Royo leg.) (MNCN). Hontoria: 8-VII-1977, 1 ejemplar (C. Rey leg.) (MNCN). Silos: 3 ejemplares (P. Saturio leg.) (MNCN). CÁCERES: El Borbollón: 21-IV-1981, 4 ejemplares (F. M. Piera leg.) (MNCN). Río Ruecas, Zorita: 25-IV-1998, 3 ejemplares (E. Pérez Zapirain leg.) (MNCN). Sierra de Guadalupe: IV-1903, 1 ejemplar (MNCN). Torrequemada: V-1935, 14 ejemplares (Gil Collado leg.) (MNCN). Trujillo-Puerto de Miravete: 1-VI1986, 1 ejemplar (A. Zuzarte leg.) (Col. A. Zuzarte). CÁDIZ: Jerez: 1 ejemplar (MNCN); IV-1908, 1 ejemplar (E. Pons leg.) (MNCN). Puerto Real: III-1919, 1 ejemplar (Smith leg.) (MNCN). San Fernando: IX-1934, 1 ejemplar (MNCN). Sierra Carbonera: 3 ejemplares (MNCN). CIUDAD REAL: Alcázar: 3 ejemplares (Escalera leg.) (MNCN). Almodóvar del Campo: 18-IV-1957, 1 ejemplar (G. Ochoa leg.) (MNCN). Anchuras, Finca Rosalejo: 25-III-1997, 2 ejemplares (M. París y E. Izquierdo leg.) (MNCN). Ciudad Real: 10-IV-1996, 1 ejemplar (L. Beugada leg.) UA). Herrera - Manzanares: V-1909, 1 ejemplar (Expedición del Museo) (MNCN). Saceruela: 1 ejemplar (MNCN). Solana del Pino, Río Robledillo-Río Frío : 20-IV1997, 3 ejemplares (M. París y E. Izquierdo leg.) (MNCN). CÓRDOBA: Cabra: 3 ejemplares (J. Carandell leg.) (MNCN). Valsequillo: 3 ejemplares (MNCN). CUENCA: $2 \mathrm{Km}$ al E de 
Arcos de la Sierra: 18-V-1998, 1 ejemplar (M. García París y C. Martín leg.) (MNCN). $4 \mathrm{Km}$ al SE de Arcos de la Sierra: 18V-1998, 2 ejemplares (M. García París y C. Martín leg.) (MNCN). Cuenca: 3 ejemplares (MNCN); VI, 2 ejemplares (E. Morales leg.) (MNCN); 20-VII-1995, 1 ejemplar (E. Camuñas leg.) (UA). La Ceja, $10 \mathrm{Km}$ al O de Las Majadas: 11-VII-1998, 1 ejemplar (M. García París y C. Martín leg.) (MNCN). Las Torcas, 4 Km NE de Mohorte: 17-V-1998, 1 ejemplar (M. García París y C. Martín leg.) (MNCN). Nacimiento del Río Cuervo: 7-V-1983, 1 ejemplar (M. Ortego leg.) (Col. A Zuzarte). $3 \mathrm{Km}$ al S de Portilla: 18-V-1998, 1 ejemplar (M. García París y C. Martín leg.) (MNCN). $2 \mathrm{Km}$ al E de Ribatajada: 18-V-1998, 1 ejemplar (M. García París y C. Martín leg.) (MNCN). $2 \mathrm{Km}$ al NO de Ribatajadilla: 18-V1998, 1 ejemplar (M. García París y C. Martín leg.) (MNCN). Tragacete: VII-1906, 2 ejemplares (Arias leg.) (MNCN). GRANADA: Almacilas, Sierra de Segura: 1 ejemplar (J. Cabré leg.) (MNCN). Cúllar de Baza: 1 ejemplar (MNCN). Granada, Cerro del Sol, Alhambra: 1 ejemplar (Aranzadi) (MNCN). Güejar Sierra: 1 ejemplar (MNCN). Pinos Puente: IV-1909, 1 ejemplar (Expedición del Museo) (MNCN). Santiesteban, Sierra Sagra: 1 ejemplar (J. Cabré leg.) (MNCN). Sierra de la Sagra: 30-IV-1994, 1 ejemplar (E. Micó leg.) (UA). Sierra Nevada: VII-1903, 10 ejemplares (Escalera leg.) (MNCN). Sierra Nevada, Lomo del Tfno [Telégrafo]: 17-VII-1972, 1 ejemplar (A. Machado leg.) (MNCN). Sierra Nevada, Veleta: 27-VI-1962, 1 ejemplar (MNCN). GUADALAJARA: $1 \mathrm{Km}$ al S de Chiloeches: 15-V-1998, 1 ejemplar (M. García París leg.) (MNCN). $3 \mathrm{Km}$ al S de Chiloeches: 15-V-1998, restos de 1 ejemplar (M. García París y C. Martín leg.) (MNCN). El Vado: 9-IV-1973, 1 ejemplar (MNCN). HUELVA: Cala: 3 ejemplares (MNCN); 1 ejemplar (C. Bolívar leg.) (MNCN). Paterna del Campo: IV-1985, 1 ejemplar (I. Izquierdo leg.) (MNCN) Tarsis: V-1908, 3 ejemplares (Expedición del Museo) (MNCN). HUESCA: Embún: V-1952, 1 ejemplar (L. Báguena leg.) (MNCN). Jaca: 1 ejemplar (MNCN). Jaca, Peña Oroel: V1952, 1 ejemplar (L. Báguena leg.) (MNCN). JAÉN: Aldeaquemada: 25-IV-1992, 1 ejemplar (C. Martín leg.) (MNCN). Andújar: VI, 1 ejemplar (E. Morales leg.) (MNCN). Baeza: 2 ejemplares (Dantin leg.) (MNCN). Jabalcuz: 19-IV1994, 2 ejemplares (F. A. Montes leg.) (MNCN). Linares: 1 ejemplar (MNCN). Sierra de Jaén: 2 ejemplares (MNCN). MADRID: Provincia de Madrid: 2 ejemplares (M. Escalera leg.) (MNCN). Arganda: 19-V-1907, 4 ejemplares (C. Rodríguez leg.) (MNCN). $2 \mathrm{Km}$ al SE de Arganda: 10-VI1998, 1 ejemplar (M. García París y C. Martín leg.) (MNCN). $3 \mathrm{Km}$ al S de Arganda: 5-VI-1998, 1 ejemplar (M. García París) (MNCN). 4 Km al SE de Arganda: 12-V-1998, 1 ejemplar (M. García París y C. Martín leg.) (MNCN). 3 Km al NO de Camporreal: 16-V-1998, 2 ejemplares (M. García París y C. Martín leg.) (MNCN). Cercedilla: 2 ejemplares (J. Ardois leg.) $(\mathrm{MNCN}) ; 1$ ejemplar (C. Bolívar leg.) (MNCN). Chinchón: 1 ejemplar (Dusmet leg.) (MNCN). Cobeñas: 25-V-1975, 1 ejemplar (MNCN). El Molar: 1 ejemplar (MNCN). Loeches: 2 ejemplares (G. Mercet leg.) (MNCN). Madrid: 4 ejemplares (MNCN); 1 ejemplar (J. Ardois leg.) (MNCN); 1 ejemplar (Arias leg.) (MNCN); 1 ejemplar (F. Bonet leg.) (MNCN); 1 ejemplar (J. Carrasco leg.) (MNCN); 2 ejemplares (Col. Pérez Arcas) (MNCN); 7 ejemplares (A. Sanz leg.) (MNCN); 1 ejemplar (A. Zarco leg.) (MNCN); VI-1909, 2 ejemplares (Expedición del Museo) (MNCN); 23-IV-1950, 1 ejemplar (E. Pons leg.) (MNCN). Madrid, Aluche: IV-1978, 1 ejemplar (M.
García París leg.) (MNCN). Madrid, Canal [de Isabel II]: 1 ejemplar (MNCN). Madrid, Cerro Negro: 25-III-1907, 1 ejemplar (C. Rodríguez leg.) (MNCN). Madrid, Ciudad Universitaria: 21-IV-1945, 1 ejemplar (MNCN). Madrid, Dehesa de la Villa: 9-VII-1909, 1 ejemplar (C. Rodríguez leg.) (MNCN). Madrid, El Pardo: 1 ejemplar (Arias leg.) (MNCN). Madrid, Moncloa: 30-IV-1909, 1 ejemplar (C. Rodríguez leg.) (MNCN). Madrid, Santa Eugenia, Cerro Almodóvar: 20-V1998, 1 ejemplar (M. García París leg.) (MNCN). Madrid, Vallecas, Cerro Almodóvar: 15-V-1987, 1 ejemplar (M. Candela leg.) (MNCN); 23-III-1989, 1 ejemplar (F. A. Montes leg.) (MNCN). Madrid, Vallecas: 1 ejemplar (G. Carrasco leg.) (MNCN); 23-IV-1907, 1 ejemplar (C. Rodríguez leg.) (MNCN). Madrid, Vicálvaro: 4 ejemplares (G. Schramm leg.) (MNCN). Montarco: 4 ejemplares (C. Bolívar leg.) (MNCN). Montejo de la Sierra: 6 ejemplares (C. Bolívar leg.) (MNCN); 9-VII-1907, 1 ejemplar (C. Rodríguez leg.) (MNCN). Moralzarzal: 1 ejemplar (MNCN). Paracuellos del Jarama: VI1935, 4 ejemplares (B. Quirós leg.) (MNCN). Ribas: 10 ejemplares (Bolívar leg.) (MNCN). Rivas: 1 ejemplar (Dusmet leg.) (MNCN). Sierra de Guadarrama: 1 ejemplar (J. Lauffer leg.) (MNCN). Soto del Real-Colmenar Viejo: 6-VI-1985, 2 ejemplares (MNCN). Vaciamadrid: V-1956, 1 ejemplar (E. Ortiz leg.) (MNCN). Villaverde: 10-VI, 1 ejemplar (C. Rodríguez leg.) (MNCN). MÁLAGA: El Pilar, Cuevas [de] San Marcos: 16-IV-1977, 1 ejemplar (M. Alonso-Zarazaga leg.) (MNCN). Málaga: 1 ejemplar (A. Sanz leg.) (MNCN). MURCIA: Jumilla: 5-VI-1994, 1 ejemplar (M. C. Mira leg.) (UA). SALAMANCA: Béjar: 960 m, VII-1954, 3 ejemplares (Expedición Instituto Español de Entomología) (MNCN). Negrilla: 7 ejemplares (C. Escribano leg.) (MNCN). SEGOVIA: San Rafael: 1 ejemplar (C. Bolívar leg.) (MNCN). SORIA: Castillo de Medinaceli: 7-VI-1997, 1 ejemplar (J. París y M. García Rollán leg.) (MNCN). Soria: 2 ejemplares (M. Loro leg.) (MNCN). TARRAGONA: Capsanes: 21-II-1981, 2 ejemplares (J. y E. Vives leg.) (Col. A. Zuzarte). TERUEL: Bronchales: VII-1956, 2 ejemplares (Expedición del Instituto Español de Entomología) (MNCN). Cedrilla: 1 ejemplar (F. Gómez Llueca leg.) (MNCN). $4 \mathrm{Km}$ al SO de Peñarroya de Tastavins: 19-VI1998, 1 ejemplar (M. García París y C. Martín leg.) (MNCN). Teruel: 3 ejemplares (A. Sanz leg.) (MNCN); V-1930, 1 ejemplar (B. Muñoz leg.) (MNCN); VI-1931, 1 ejemplar (B. Muñoz leg.) (MNCN); 5-V-1934, 1 ejemplar (B. Muñoz leg.) (MNCN). TOLEDO: Erustes: VI-1943, 1 ejemplar (E. Pons leg.) (MNCN). Quero: V-1908, 2 ejemplares (J. Sanz leg.) (MNCN). Toledo: 1 ejemplar (G. Menor leg.) (MNCN). VALENCIA: Burjasot: 22-X-1990, 1 ejemplar (UA). Onteniente: 20-IV-1995, 2 ejemplares (Manresa leg.) (UA). Valencia: 2 ejemplares (Lruz Nathan leg.) (MNCN). Villamarchante: 1 ejemplar (Moroder leg.) (MNCN). VALLADOLID: Alaejos: 1 ejemplar (MNCN). VIZCAYA: 4 ejemplares (C. Cardin leg.) (MNCN). ZAMORA: Argujillo: 9-V-1998, 1 ejemplar (M. García París y C. Martín leg.) (MNCN). 3 Km al NO de Guarrate: 9-V-1998, 1 ejemplar (M. García París y C. Martín leg.) (MNCN). PORTUGAL: ALGARVE: Barragem da Bravura: 15-V-1982, 1 ejemplar (A. Zuzarte leg.) (Col. A. Zuzarte). Lagos: V-1909, 1 ejemplar (Expedición del Museo) (MNCN). San Marcos da Serra: V-1909, 2 ejemplares (Expedición del Museo) (MNCN). Sierra de Monchique, Foia: 20-IV-1982, 4 ejemplares (T. Branco leg.) (Col. A. Zuzarte). Sierra de Monchique: 25-IV-1978, 1 ejemplar (A. Zuzarte leg.) (Col. A. Zuzarte). ALTO ALENTEJO: Elvas-Caia: 21-III- 
1989, 1 ejemplar (A. Zuzarte leg.) (Col. A. Zuzarte). Evora, Estrada de Montemor, a $8 \mathrm{Km}$ de Evora: 22-V-1980, 1 ejemplar (A. Zuzarte y E. Luna leg.) (Col. A. Zuzarte). S. Cristobão, $\mathrm{EN} \mathrm{n}^{\circ}$ 253, Km 57: 9-IV-1982, 6 ejemplares (A. Zuzarte leg.) (Col. A. Zuzarte). St. Amaro-Sousel: 2-IV-1977, 2 ejemplares (A. Zuzarte leg.) (Col. A. Zuzarte); 21-IV-1968, 1 ejemplar (A. Zuzarte leg.) (Col. A. Zuzarte). Val das Quintas-Veiros: 31-III1968, 1 ejemplar (A. Zuzarte leg.) (Col. A. Zuzarte); 3-IV1986, 1 ejemplar (A. Zuzarte leg.) (Col. A. Zuzarte). BAIXO ALENTEJO: Beja: V-1909, 2 ejemplares (Expedición del Museo) (MNCN). Beja-Neves: 3-V-1983, 1 ejemplar (A. Zuzarte leg.) (Col. A. Zuzarte). Vilaverde de Ficalho: 9-IV1983, 3 ejemplares (T. Branco leg.) (Col. A. Zuzarte). BEIRA ALTA: Sabugal: 6-VII-1981, 1 ejemplar (A. Zuzarte leg.) (Col A. Zuzarte). RIBATEJO: Coruche-Bairro do Serração: 26-III-
1977, 2 ejemplares (A. Zuzarte leg.) (Col. A. Zuzarte). Foros de Benfica: 30-V-1982, 4 ejemplares (A. Zuzarte leg.) (Col. A. Zuzarte). Muge: 13-V-1967, 5 ejemplares (T. Branco leg.) (Col. A. Zuzarte). TRAS-OS-MONTES E ALTO DOURO: Bragança-Rebordainhos: 11-VI-1983, 2 ejemplares (A. Zuzarte leg.) (Col. A. Zuzarte). Serra de Bornes: 26-IV-1984, 2 ejemplares (T. Branco leg.) (Col. A. Zuzarte). 